\title{
CORRELAÇÃO ENTRE MARCADORES DE VULNERABILIDADE SOCIAL FRENTE AO USO DO PRESERVATIVO POR TRABALHADORAS SEXUAIS
}

\section{Pablo Luiz Santos Couto}

Mestre em Enfermagem. Docente do Colegiado de Enfermagem do Centro de Ensino Superior de Guanambi, Guanambi, BA, Brasil.

\section{Antônio Marcos Tosoli Gomes}

Enfermeiro. Doutor em Enfermagem. Docente do Departamento de Enfermagem Médico Cirúrgica e do Programada de Pós-Graduação em Enfermagem da Universidade do Estado do Rio de Janeiro, Brasil.

\section{Alacoque Lorenzini Erdmann}

Enfermeira. Doutora em Enfermagem. Docente Titular da Universidade Federal de Santa Catarina, SC, Brasil.

\section{Ozeli Oliveira Brito}

Doutora em Enfermagem. Enfermeira do Colegiado de Enfermagem do Centro de Ensino Superior de Guanambi, Guanambi, BA, Brasil.

\section{Virgínia Paiva Figueiredo Nogueira}

Enfermeira. Doutora em Enfermagem. Pósdoutoranda pelo Programa de Pós-Graduação em Enfermagem da Universidade do Estado do Rio de Janeiro, RJ, Brasil.

\section{Carle Porcino}

Psicologa. Mestra em Estudos Interdisciplinares da Universidade. Doutoranda no Programa de PósGraduação em Enfermagem e Saúde (PPGNEF)/Escola de Enfermagem da Universidade Federal da Bahia, Brasil.

\section{Alba Benemérita Alves Vilela}

Enfermeira. Doutora em Enfermagem. Docente do Departamento de Saúde da Universidade Estadual do Sudoeste da Bahia, Brasil.
RESUMO: Objetivou-se analisar a correlação entre os marcadores de vulnerabilidade social com o uso do preservativo entre trabalhadoras sexuais. Estudo quantitativo, inferencial e de corte transversal realizado com 138 mulheres no sertão produtivo da Bahia, em abril de 2017. Foi utilizado o software SPSS, versão 22.0, para a realização dos testes estatísticos $r$ de correlação, $p$ de Pearson e Teste-t student; adotou-se o nível de significância de 5\%. O teste $\mathrm{r}$ apontou correlação do uso de preservativo entre as mulheres com idade, cor autodeclarada e o tempo de serviço. No teste $p$ de Pearson, a idade relacionada ao uso do preservativo, o resultado foi negativo, o Teste-t resultou em duas amostras $\mathrm{r}$ e p com variâncias equivalentes. As três correlações estabelecidas entre os marcadores de vulnerabilidade social e o uso de preservativo pelas trabalhadoras sexuais são significantes; ressaltase que o nível de escolaridade não influenciou na adesão ao uso do preservativo.

PALAVRAS-CHAVE: Profissionais do sexo; Vulnerabilidade social; Saúde sexual; Saúde pública; Promoção da saúde.

\section{CO-RELATIONSHIP BET WEEN SOCIAL VULNERABILITY Y MARKERS AND THE USE OF CONDOMS BY SEX WORKERS}

\begin{abstract}
The co-relationship between social vulnerability markers and the use of condoms among sex workers is provided through a quantitative, inferential and transversal research with 138 females within the production sector of Bahia, Brazil, during April 2017. SPSS 22.0 was employed for co-relation statistic tests $r$, Pearson's $p$ and Student's t test, at 5\% significance. Test $r$ indicated a co-relationship in the use of condoms by females according to age, color and service time. In the case of Pearson's test, there was a negative result in age related to the use of condoms, whilst test $t$ provided two samples $r$ and $\mathrm{p}$ with equivalent variables. Established co-relationships between social vulnerability markers and the use of condoms by sexual workers were significant. Schooling level did not affect adhesion to the use of condoms.
\end{abstract}

KEY WORDS: Sex Workers; Social Vulnerability; Sexual Health; Public Health; Health Promotion.

\section{INTRODUÇÃO}

A prática da prostituição é uma temática polêmica, antiga, e carregada de tabus e estigmas, porém, ainda atual e cheia de prós e contras, de instabilidades e não afeito à cultura, mesmo já existindo uma lei que visa à legalização da profissão, no intuito de torná-la menos 


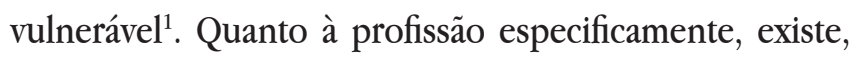
no Brasil, um Projeto de Lei (PL), de 2012, denomado Gabriela Leite que tramita no Congresso Nacional e regulamenta a atividade das pessoas profissionais do sexo. Segundo o artigo $1^{\circ}$ do texto, pode-se depreender que é profissional do sexo toda pessoa maior de 18 anos e totalmente capaz que, espontaneamente, decida prestar serviços sexuais consoante pagamento ${ }^{2}$.

Entende-se que a vulnerabilidade não é específica de uma pessoa ou grupo específico, mas daqueles que se encontram em situações de vida que podem ser melhoradas. No caso das profissionais do sexo em questão, é a condição a qual estas mulheres estão expostas que as vulnerabilizam, com tendência nos três aspectos: pessoal, programático ou social, o que torna difícil seu enfrentamento de vida $a^{3-5}$.

No nível pessoal a vulnerabilidade está ligada às consequências do comportamento do indivíduo que o expõe a condições que podem levá-lo a infectar-se ou a adoecer por meio dos danos causados diretamente em si. Já no nível programático, o que está em foco são as políticas e ações criadas para tentar evitar os conflitos advindos das Infecções Sexualmente Transmissíveis (IST's)/Síndrome da Imunodeficiência Adquirida (AIDS) 5 . O plano social relaciona-se a fatores sociodemográficos, escolaridade, religiosos, culturais, comunitários, questões de gênero e na vontade dos grupos de profissionais e das pessoas de realizar ações de promoção, prevenção e proteção das IST's, sendo tais fatores identificados como marcadores de vulnerabilidade social ${ }^{6}$.

Os marcadores de vulnerabilidade são dados importantes que revelam o nível de estigmatização e marginalização social de grupos e foi conceituado em um estudo desenvolvido na Malásia sobre identidade de gênero e acesso aos serviços de saúde ${ }^{7}$. Tais marcadores estão relacionados às condições sociodemográficas, as quais possibilitam o levantamento de informação sobre as características da população e tudo o que possa levála à essa condição, que poderão proporcionar, mediante análise por parte da sociedade e dos profissionais de saúde, o planejamento de ações visando a compreensão e a superação das vulnerabilidades ${ }^{5,8}$.

As trabalhadoras sexuais encontram-se vulneráveis pelas situações que enfrentam no cotidiano da profissão, dentre as quais pode-se citar, o baixo nível socioeconômico e de escolaridade e o medo de procurar os serviços de saúde e sofrer estigma e preconceito 9 . Além destes pode-se incluir, ainda, a violência de clientes que, para satisfazerem-se, praticam atos agressivos obrigandoas em muitos momentos às atividades sexuais forçadas $\mathrm{e}$ desumanas que levam ao medo e causam danos físicos e psicológicos. No Canadá, por exemplo, a suprema corte teve que intervir ao descriminalizar o trabalho sexual, pois a violência sexual contra as trabalhadoras sexuais estava alcançando dados alarmantes com associação direta ao aumento da infecção pelo HIV ${ }^{10}$. Destacam-se, também, múltiplos parceiros, negação de alguns clientes do uso do preservativo, o que aumenta a probabilidade da transmissão das IST/AIDS e o envolvimento com álcool e drogas ${ }^{4}$.

O contexto da prevenção ao HIV entre trabalhadoras sexuais no Brasil continua sendo um desafio em análise de desenvolvimento de novas estratégias de prevenção. Decorrido três décadas como foco de campanhas de prevenção entre as profissionais do sexo, continuam a ter uma prevalência de HIV de 4,9\% estimada em mais de 15 vezes maior do que a prevalência na população geral de mulheres entre 15 e 49 anos. ${ }^{11}$ Em 110 países com dados disponíveis, a prevalência de infecção pelo HIV é quase 12 vezes maior entre trabalhadoras sexuais do que para a população como um todo, com prevalência pelo menos 50 vezes maior em subdesenvolvidos e regiões pobres ao redor do mundo ${ }^{12}$ não apenas pelo fato individual/comportamental (volume e simultaneidade de parceiros e uso de drogas injetáveis), mas, sobretudo, por questões estruturais e sociais, como a organização do trabalho sexual, pobreza, baixo nível de escolaridade, idade, habitação, insegurança financeira, políticas legais e de criminalização e violência ${ }^{13}$.

Enquanto um estudo evidenciou que tem sido implementado na América Latina políticas de apoio à saúde sexual, reprodutiva e de prevenção à epidemia da aids entre mulheres profissionais do sexo, revelando que, nas últimas duas décadas, houve um aumento no uso de preservativos entre elas ${ }^{14}$, em outro foi registrado que muitas trabalhadoras sexuais sofrem pressão por parte de alguns clientes que alugam seus corpos para realizar o sexo sem preservativo9 ${ }^{9}$. Este presente estudo aponta 
possibilidades de olhar para um grupo populacional vulnerável socialmente, de uma região carente do país, o semiárido do nordeste brasileiro, sobretudo no que se refere à saúde sexual e à prevenção de agravos como as IST's por meio do uso do preservativo ${ }^{4,6}$.

Diante o exposto, questiona se existe correlação entre os marcadores de vulnerabilidade social e à adesão ou não ao uso do preservativo entre trabalhadoras sexuais de uma região carente dos país? Destarte, tem-se como objetivo analisar a correlação entre os marcadores de vulnerabilidade social com o uso do preservativo entre as trabalhadoras sexuais. A hipótese deste estudo foi há correlação entre os marcadores idade, nível de escolaridade, cor autodeclarada e tempo de serviço para a adesão ao uso do preservativo entre as trabalhadoras sexuais.

\section{METODOLOGIA}

Tratou-se de um estudo de corte transversal e inferencial, com abordagem quantitativa e que se aportou em cálculos de recursos bioestatísticos. O cenário do estudo foi a mesorregião de Guanambi-BA, sede do Alto Sertão Produtivo Baiano, que abrange cerca de 19 municípios, com pouco mais de 400.000 habitantes ${ }^{15}$, nas áreas de vulnerabilidade social, onde se encontram os locais de trabalho das profissionais do sexo.

A amostra da pesquisa foi composta por 138 mulheres, sendo uma amostra por conveniência, uma vez que se trata de um grupo que tem invisibilidade social, com poucos registros de indicadores do quantitativo delas na região e no país. Adotou-se como critérios de inclusão mulheres profissionais do sexo com idade igual ou superior a 18 anos que mantém vida sexual ativa, em uso, ou não de preservativo nas relações sexuais. Não houve a aplicabilidade de critérios de exclusão, visto que só contribuíram com o estudo aquelas que aceitaram o convite para participação.

A aproximação com as mulheres foi feita por meio do Centro de Testagem e Aconselhamento (CTA) e das agentes de saúde. O CTA atua dentro desta área, o que facilitou a aproximação da equipe com estas mulheres, reiterando-se que muitas delas têm forte adesão aos programas de prevenção do CTA, pois existe no município uma forte campanha de intervenção com elas, elaboradas junto ao Ministério da Saúde. A coleta de dados ocorreu individualmente entre os meses de abril de 2017 a março de 2018, em salas fechadas nas Estratégias de Saúde da Família dos bairros em que estão localizados os vários estabelecimentos em que as profissionais do sexo trabalham. Contudo, algumas mulheres não se disponibilizaram a se deslocar e, por este motivo, foi agendada uma visita ao local de trabalho junto ao agente comunitário da área, onde também foram realizadas as coletas de informações.

Para a coleta de dados foi aplicado um formulário pelos pesquisadores, composto de questões que englobaram os dados socioeconômicos que compuseram as questões referentes à vulnerabilidade social, além de questões sobre a vivência do cotidiano da profissão, doenças associadas e aquelas referentes à saúde sexual e reprodutiva. Para este estudo foram trabalhados a correlação dos marcadores de vulnerabilidade social (sociodemográficos) com o uso de preservativo.

Para a análise dos marcadores de vulnerabilidade social que fazem correlação à adesão ao uso ou não do preservativo entre as participantes estabeleceramse as seguintes variáveis: idade, escolaridade, cor autodeclarada, religião, tempo de serviço, satisfação com a profissão e uso ou não de preservativo.

Foram utilizados os softwares Microsoft Excel 2016, para organização e categorização e dados, e o Statistical Package for the Social Sciences - SPSS versão 22.0 de 2013, responsável por proceder com os testes estatísticos adotados para este estudo. Os testes $r$ de correlação e $p$ de Pearson foram utilizados para verificar a correlação entre as variáveis, independente e dependente, adotando-se o nível de significância de 0,05 e intervalo de confiança de $95 \%$. O teste de correlação $r$ verificou se houve correlação entre as variáveis $\mathrm{X}$ e $\mathrm{Y}$. $O$ teste $p$ de Pearson serviu para validar a correlação do teste $r$ e explicar como esta correlação se comportou. Quando foi feita a comparação avaliou-se se $r$ e $p$ foram maiores ou menores que o nível de significância de 0,05 . Quando se mostraram maior que 0,05 entende-se que não houve correlação entre as variáveis, aceitando, portanto, a hipótese nula. Se ele foi menor que 0,05 dizse que houve correlação entre as variáveis, aceitando-se a hipótese alternativa. 
Após a aplicação de ambos os testes de correlação $r$ e $p$, os resultados foram pareados e colocados à prova sob o teste $t$ Student, com o intuito de verificar se as correlações evidenciadas poderiam ser validadas estatisticamente. Para tanto, considerou-se como parâmetro para aceitar as correlações (H0) ou rejeitá-las (HA), o valor de " $\mathrm{P}(\mathrm{T}<=\mathrm{t})$ uni-caudal" maior que valor de significância ou " $\mathrm{P}(\mathrm{T}<=\mathrm{t})$ uni-caudal" menor que nível de significância, respectivamente.

A pesquisa foi realizada de acordo aos princípios éticos da resolução no 466/2012 do Conselho Nacional de Saúde, o qual foi submetido ao CEP - Comitê de Ética em Pesquisa, em seguida foi aprovada com protocolo número 2.007.080/2017. Os aspectos éticos discutidos na resolução que têm como finalidade garantir o sigilo e anonimato das participantes, visando autonomia, beneficência e dignidade foram observados. Todas as participantes assinaram um Termo de Consentimento Livre e Esclarecido (TCLE).

\section{RESULTADOS}

Entre as 138 mulheres incluídas nesta pesquisa, de acordo com as variáveis sociodemográficas da amostra adaptadas como marcadores de vulnerabilidade social do estudo e, conforme percebido na Tabela 1 , a maioria das mulheres (78,26\%) tinha entre 18 e 35 anos de idade e possuía nível fundamental $(53,62 \%)$; também a maioria delas se declarou negras $(59,42 \%)$ e católicas $(55,07 \%)$. Quanto ao tempo de serviço os sujeitos relatam trabalhar há menos de cinco anos (68,12\%), a maioria (55,97\%) não estava satisfeita com a profissão e cerca de $63,77 \%$ relataram usar preservativos durante as relações sexuais.

Tabela 1. Características sociodemográficas adaptadas como marcadoras de vulnerabilidade social das mulheres profissionais do sexo que usam ou não usam preservativos. Guanambi, BA, Brasil, 2017

(Continua)

\begin{tabular}{lcc}
\hline VARIÁVEIS & $\mathbf{n}$ & (\%) \\
\hline IDADE & & \\
$\quad 1$ (18 a 35 anos) & 108 & $(78,26)$ \\
2 (35 ou mais) & 30 & $(21,74)$ \\
NÍVEL DE ESCOLARIDADE & & \\
$\quad$ (Fundamental) & 74 & $(53,62)$ \\
2 (Médio) & 64 & $(46,38)$
\end{tabular}

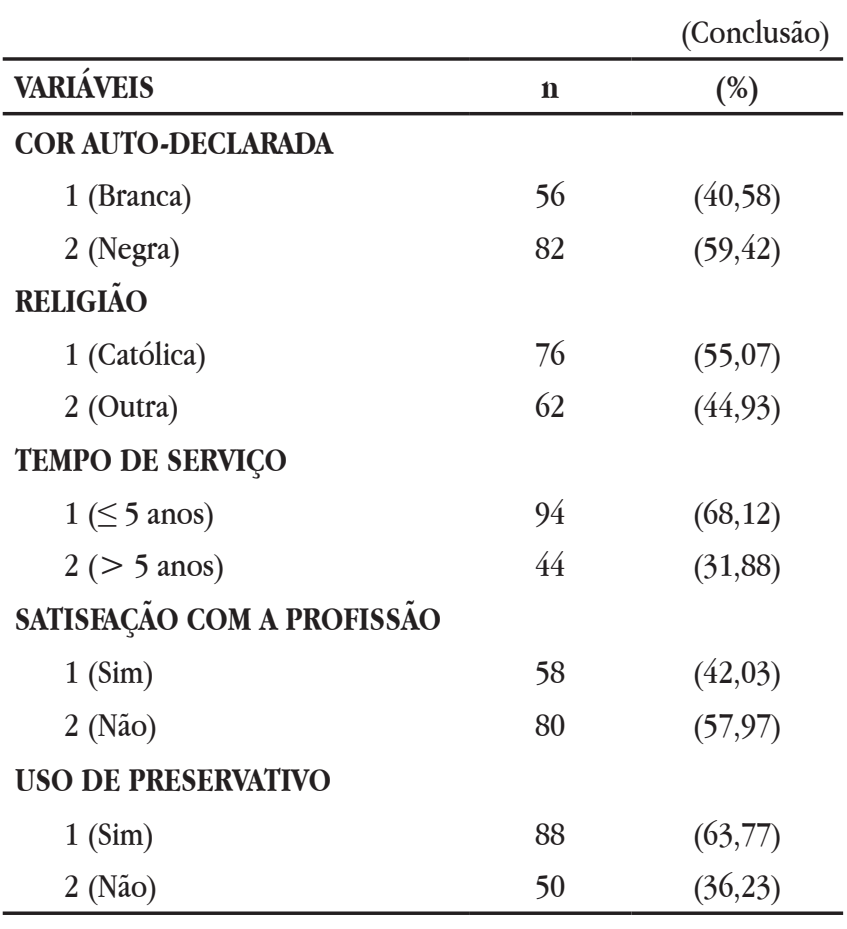

O teste $r$ apontou correlação do uso de preservativo entre as mulheres com as variáveis: idade, cor autodeclarada e o tempo de serviço, sendo validada a correlação com o teste $p$ de Pearson (Tabela 02). Estes três marcadores apresentaram correlação com o uso do preservativo, uma vez que apresentaram valor menor que o nível de significância 0,05 , rejeitando-se a hipótese nula. Os demais marcadores não tiveram correlação com o uso do preservativo para este estudo porque o teste $r$ de correlação apresentou valor maior que o nível de significância 0,05 , confirmado pelo teste $p$, assim, inferese que não houve correlação entre os três porque foi aceita a hipótese nula.

Conforme se observa ainda na Tabela 2 o teste $p$ para as variáveis, idade com o uso do preservativo ele apresentou resultado negativo, ou seja, ele quer mostrar que à medida que as mulheres envelhecem aumenta o não uso do preservativo. Para a cor autodeclarada houve correlação estatisticamente significante uma vez que teste $r$ foi menor que 0,05 tendo sido validado pelo teste $p$ que apresentou o mesmo resultado. Contudo, $p$ e $r$ foram positivos, pois à medida que aumenta o número de mulheres negras a frequência do uso de preservativo aumenta, o que demonstra um resultado óbvio, visto que a maior parte da população do estudo é de mulheres negras. 
Tabela 2. Tabela de correlações entre os marcadores de vulnerabilidade social e o uso de preservativos. Guanambi, BA, Brasil, 2017

\begin{tabular}{lccc}
\hline & & USO DE PRESERVATIVOS \\
\hline Marcadores de vulnerabilidades & Teste $\boldsymbol{r}$ de correlação & Teste $\boldsymbol{p}$ de Pearson & Nível de significância $\mathbf{0 , 0 5}$ \\
\hline Idade & $-0,02131$ & $-0,02131$ & Teste $\mathrm{p}<0,05$ \\
Nível de escolaridade & $-0,0963$ & $-0,0963$ & Teste $\mathrm{p}>0,05$ \\
Cor auto declarada & 0,008899 & 0,008899 & Teste $\mathrm{p}<0,05$ \\
Religião & $-0,13529$ & $-0,13529$ & Teste $\mathrm{p}>0,05$ \\
Tempo de serviço & 0,001875 & 0,001875 & Teste $\mathrm{p}<0,05$ \\
Satisfação com a profissão & $-0,21335$ & $-0,21335$ & Teste $\mathrm{p}>0,05$ \\
\hline
\end{tabular}

No intuito de verificar se as correlações existentes, apresentadas na Tabela 02, são estatisticamente aceitáveis, procedeu-se com o Teste-t student para prova de normalidade, presumindo-se duas amostras (testes $r$ e $p$ ) com variâncias equivalentes. Desse modo, as três correlações estabelecidas entre os marcadores de vulnerabilidade social (idade, cor autodeclarada e tempo de serviço) com o uso de preservativo pelas mulheres profissionais do sexo são significantes, visto que os valores de $\mathrm{P}(\mathrm{T}<=\mathrm{t})$ unicaudal $(=0,5)$ e $\mathrm{P}(\mathrm{T}<=\mathrm{t})$ bicaudal $(=1)$ foram maiores que o nível de significância $(0,05)$, respectivamente: $t$ crítico unicaudal igual a 1,859 e t crítico bicaudal igual a 2,306.

\section{DISCUSSÃO}

A prostituição é regulamentada pelo Ministério do Trabalho por meio da (Classificação Brasileira de Ocupações) CBO 5198 - 05, que apresenta normas para que as mulheres profissionais do sexo exerçam a profissão ainda que sejam vulneráveis socialmente: trabalhar aonde tiver chance, mas por conta própria, profissão oficialmente permitida para maiores de 18 anos de idade. ${ }^{2}$ As vulnerabilidades as quais estão expostas tem seu início desde cedo: algumas entram para o serviço aos 12 anos de idade e, em função da idade, sentem medo do ambiente onde estão inseridas, sobretudo quanto à falta do uso do preservativo. A maioria relatou o início da vida profissional muito cedo e sofre rejeição por parte de familiares, o que são agravantes ${ }^{3}$.

Em um estudo internacional de revisão de literatura sobre determinantes da prevalência e incidência do HIV e o uso do preservativo entre trabalhadoras sexuais ao redor do mundo, evidenciou que a idade é um dos determinantes para a não adesão ao preservativo, contudo isso foi perceptível em países subdesenvolvidos e entre as menos instruídas ${ }^{10}$. O abandono da escola e a prostituição são uma relação que deve ser aprofundada e analisada em diferentes grupos sociais, uma vez que a maioria das mulheres que se prostitui só conclui o ensino fundamental. Algumas delas relatam que é muito difícil associar a escola com o seu trabalho, pois o trabalho envolve a noite, turno preferido pelos seus usuários. Durante o dia procuram outros serviços para aumentar a sua renda financeira, outras vão dormir para recuperar o turno de sono perdido, outras não suportariam o olhar de desprezo advindo da sociedade estudantil e, com isto, a escola acaba por ficar em segundo plano ou deixa de existir em seus planos ${ }^{16}$.

É necessário destacar, que para este presente estudo, o nível de escolaridade não apresentou correlação com o uso de preservativo, ou seja, não interferiu na adesão das mulheres profissionais do sexo à camisinha, o que difere de alguns estudos desenvolvidos no Brasil e no mundo ${ }^{12,14,16-17}$. Porém, esta característica será percebida em diferentes pesquisas, pois os aspectos da prostituição modificam conforme as localidades e o acesso às políticas públicas de saúde ${ }^{11}$. Ainda que muitas dessas mulheres, que tem no aluguel do seus corpos a fonte de renda, não avançam no nível de escolaridade, o conhecimento transmitido por meio das atividades desenvolvidas nas unidades de saúde, por exemplo, no âmbito dos programas e políticas públicas de prevenção ao HIV, é fundamental que esse grupo de mulheres vulneráveis 
tenham adesão ao uso do preservativo durantes as práticas sexuais $^{17-18}$.

A cor autodeclarada apresentou correlação estatisticamente significantes em relação ao uso de preservativos, pois as mulheres negras sofrem mais preconceitos e, de um modo geral, vivem em periferias e estão suscetíveis a mais agravos que as colocam em situações de vulnerabilidades ${ }^{19}$. Destaca-se que elas as mulheres negras, e portanto, as trabalhadoras sexuais negras, são maioria no Brasil, sendo que elas estão em maior quantidade e, por isto, são as que têm tido acesso à política de combate à epidemia da aids ${ }^{14}$. Reiterase que em decorrência da realidade escravocrata e do modo como a população negra foi se organizando nas periferias e favelas das cidades e grandes centros, sendo marginalizadas, estigmatizadas e segregadas socialmente, foi crescendo a prostituição entre as mulheres que se declaram pretas ${ }^{20-21}$.

A correlação positiva com a cor autodeclarada pode também ser explicada pela eficácia que são as políticas e atividades de prevenção entre trabalhadoras sexuais, oriundas do Ministério da Saúde, adotadas no território brasileiro conforme as equipes dos Centros de Testagem e Aconselhamento e todas as equipes da unidade básica. ${ }^{11}$ Além disso, os serviços públicos da atenção básica, cujas políticas públicas de combate à epidemia da aids são adotadas, em sua maioria, são acessados por pessoas baixo poder aquisitivo e que normalmente são da raça negra22.

Um estudo de revisão internacional, que investigou a epidemiologia do HIV entre mulheres profissionais a partir de conceitos de determinantes sociais, apresentou uma ressalva quanto a essa correlação positiva, de que fatores biológicos como raça, influencia no comportamento preventivo ao HIV, especificamente no uso de preservativo, sobretudo entre aquelas que habitam as regiões mais pobres do mundo e não têm acesso à informação ${ }^{19}$.

Ao se analisar o contexto da prostituição dois fatos são averiguados: um é que ele se baseia na diferenciação de gênero e o outro nas desigualdades socioeconômicas, étnico-raciais e geracionais. Estes dois fatos são revelados não apenas pelas profissionais do sexo, mas como verificado em outro estudo, por vários grupos populacionais vulneráveis socialmente como imigrantes etnias/raças/cores discriminadas (negros, pardos, indígenas, latinos e afrodescendentes ou oriundos do Leste Europeu e da Ásia, países considerados de primeiro mundo $)^{23}$.

A religião, ainda que seja um dispositivo social e marcador de vulnerabilidade que influencia os comportamentos e as ideias tanto das pessoas individualmente quanto da sociedade, não influenciou na correlação deste estudo, pois ainda que a maioria delas se declarasse católicas, elas não eram praticantes. Um estudo evidenciou que a religião não detém domínio sobre as trabalhadoras sexuais. A partir do momento que elas deixam o ambiente familiar de origem e ingressam na prostituição, relegam crenças de infância e aderem às religiões de matrizes africanas, pois não as rejeitam, diferentemente de outras religiões como as cristãs (católicas e evangélicas) que veem a prostituição como pecado $^{1}$.

As mulheres profissionais do sexo, que professam as religiões católica e evangélica, rompem com as regras da religião e aderem à prática da prostituição para satisfazerem seus anseios de melhora financeira, de terem autonomia sobre seus corpos e de elevação de sua estima, ainda que tenham que viver uma vida clandestina entre dois mundos, o religioso e o mundano. Mesmo que a religião católica tenha um perfil menos punitivo com seus fiéis, sua doutrina estigmatiza a sexualidade e restringe a prática sexual ao casamento e, mesmo em seu interior, para fins de procriação ${ }^{2}$.

Já o tempo de serviço, marcador adotado para este estudo, é um dos agravantes para o uso do preservativo entre as mulheres profissionais do sexo, quando o valor de $p$ para essa correlação indica que quanto mais tempo na profissão, menor o uso do preservativo entre elas. Contudo, outra pesquisa desenvolvida no município de Picos-PI afirmou que profissionais do sexo com mais tempo de prostituição aceitam facilmente o preservativo para prevenção contra IST's ${ }^{22}$, o que diverge do presente estudo. Tal divergência é esperada uma vez que estudos nacionais e internacionais apontaram que as associações do uso de preservativo a fatores socioeconômicos entre trabalhadoras sexuais, como o tempo de serviço, apresentaram contradições em várias pesquisas 
decorrentes, sobretudo, da procedência e do acesso às informações que elas têm sobre a prevenção ao HIV ${ }^{10-11,17}$.

O tempo de prestação de serviços destas mulheres não é tão longo porque as vulnerabilidades a que estão expostas dificultam a permanência na prostituição. A autoestima que algumas foram buscar nessa prática logo é diminuída, pois começam a compreender que não é fácil viver uma vida de comercialização do corpo, no qual os adoecimentos, a violência sexual e a privação de uma vida com mais exposições a agravos que as tornam vulneráveis são mais frequentes ${ }^{1}$. Para algumas dessas mulheres não há satisfação com a prostituição, para outras é o próprio lucro que as satisfazem. As mulheres que realizam esta prestação de serviço começam a compreender que não vendem o corpo e sim o alugam a um ou vários parceiros, pois ninguém passa a ser dono do outro como um escravo. Elas entendem que alugam o corpo, pois no outro dia ou no mesmo, outro usuário pode recorrer aos seus serviços e ela terá que estar disposta a servi-lo².

$\mathrm{Na}$ área da política vigente de prevenção do HIV/ Aidsé necessário estar atentoà importância do preservativo masculino e/ou feminino e discutir seus limites, investigar o uso do preservativo masculino no Brasil e elaborar críticas sobre o seu papel no novo contexto da prevenção do HIV/AIDS e considerar o papel da sexualidade e do sexo nas intervenções acertadas, além da diminuição da vulnerabilidade de infecção por HIV/AIDS. ${ }^{24}$ Em junho de 2013, o Ministério da Saúde brasileiro criou a campanha "Eu sou feliz sendo prostituta" para estimular o uso de preservativos entre o grupo, mas não foi avante, especialmente porque os grupos religiosos se levantaram contra a campanha e pela exoneração do cargo de diretor de Infecções Sexualmente Transmissíveis ${ }^{25}$.

Quanto à associação da vulnerabilidade à infecção do vírus HIV/Aids concernente ao nível de escolaridade e pobreza, estudos têm mostrado que, entre o grupo soropositivo, as mulheres apresentam maior frequência em baixa escolaridade e situação de pobreza ${ }^{12-13,19,22,26}$. Este fato torna os grupos sociais menos favorecidos, inclusive as profissionais do sexo, mais vulneráveis à disseminação do vírus do HIV possivelmente por falta de conhecimento, menor acesso aos meios de prevenção e serviços de saúde, em decorrência do preconceito e estigma por parte dos profissionais de saúde, além da dificuldade em dialogar sobre o uso do preservativo ${ }^{25-26}$.

\section{CONCLUSÃO}

Conclui-se que a idade, a cor autodeclarada e o tempo de serviço foram os marcadores de vulnerabilidade social que apresentaram correlação com a adesão ao uso do preservativo entre as mulheres profissionais do sexo. Além disto, destaca-se que diferente de estudos anteriores realizados em outras localidades, nível de escolaridade e religião foram marcadores que não interferiram no uso do preservativo entre as mulheres desse estudo. Dessa forma, aceita-se parcialmente a hipótese nula, uma vez que, o único marcador de vulnerabilidade que não influenciou na adesão ao uso de preservativo entre as trabalhadoras sexuais foi o nível de escolaridade.

Apresenta-se como limitação deste estudo, o quantitativo de mulheres profissionais do sexo participante, possivelmente em decorrência do universo de pesquisa, pois foi realizado na Alto Sertão Produtivo Baiano. Ainda que as generalizações sejam dificultadas pelas singularidades da região e pelos perfis e pelas condições de vida distintas, há a relevância nesse estudo pelo fato de que algumas correlações são singulares ao grupo estudado, como as estabelecidas com nível de escolaridade e religião, além do ineditismo em abordar essa proposta de estudo (vulnerabilidade social versus adesão ao uso ou não do preservativo entre trabalhadoras sexuais).

Dentre as ações que os profissionais de saúde podem adotar, a partir da contribuição desse estudo (favorecer a discussão sobre populações vulneráveis e saúde sexual) destacam-se as intervenções de medidas de promoções à saúde voltadas para as minorias e os grupos socialmente invisíveis, como oficinas de educação em saúde, possibilitando às profissionais do sexo maior autonomia sobre si na busca pela melhoria das condições de saúde, que inevitavelmente refletirá na saúde sexual.

\section{REFERÊNCIAS}

1. Lopes N. "Prostituição sagrada" e a prostituição como objeto preferencial de conversão dos crentes. Relig Soc [Internet]. 2017 [citado 2018 set. 15];37(1):34-46. DOI: http://dx.doi. org/10.1590/0100-85872017v37n1cap02. 
2. Pereira JB, Feijó MEV. Prostituição e preconceito: uma análise do projeto de lei Gabriela Leite e a violação da dignidade da pessoa humana. Cadernos Grad [Internet]. 2014 [citado 2018 set. 15];2(1):3957. Disponível em: https://periodicos.set.edu.br/ index.php/fitshumanas/article/viewFile/1348/796.

3. Sousa PKR, Miranda KCL, Franco AC.

Vulnerabilidade: análise do conceito na prática clínica do enfermeiro vulnerabilidade: análise do conceito na prática clínica do enfermeiro em ambulatório de HIV/AIDS. Rev Bras Enferm [Internet]. 2011 [citado 2018 set. 15];64(2):3814. Disponível em: http://www.scielo.br/pdf/reben/ v64n2/a26v64n2.pdf.

4. Soares JFS, Santos LC, Cardoso JP, Neves L, Batista EC. A prostituição como profissão: uma análise sob a ótica das profissionais do sexo. Rev Saberes [Internet]. 2015 [citado 2018 set. 04];3(2):63-75. Disponível em: http:/facsaopaulo.edu.br/media/ files/35/35_1385.pdf.

5. Brisson J. Reflections on the history of bareback sex through ethnography: the works of subjectivity and PrEP. Anthropology \& Medicine [Internet]. 2017 [citado 2018 set. 04]:1-15. DOI: https://doi.org/10.10 80/13648470.2017.1365430.

6. Muñoz LA, Sanchez X, Arcos E, Vollrath A, Bonatti C. The motherhood experience in the context of social vulnerability: a comprehensive approach to social phenomenology. Rev Latino-Am Enfermagem [Internet]. 2013 [citado 2018 set. 04];21(4):9139. DOI: http://dx.doi.org/10.1590/S010411692013000400012.

7. Gibson AB, Brown SE, Rutledge R, Wickersham JA, Kamarulzaman A, Altice FL. Gender identity, healthcare access, and risk reduction among Malaysia's mak nyah Community. Glob Public Health [Internet]. 2016 [citado 2018 set. 04];11(7-8):101025. DOI: http://dx.doi.org/10.1080/17441692.2015.1 134614.

8. Chaves ACP, Bezerra EO, Pereira MLD, Wolfgang W. Conhecimentos e atitudes de adolescentes de uma escolar pública sobre a transmissão sexual do HIV. Rev Bras Enferm [Internet]. 2014 [citado 2018 set. 04];67(1):48-53. DOI: http://dx.doi. org/10.5935/0034-7167.20140006.

9. Valdés MCV, Hernández EY, Peguero MMG, Prieto
MR. Mujer posmenpáusica. ¿Fin de la vida sexual? Rev Haban Cienc Méd [Internet]. 2013 [citado 2017 oct. 07];12(2): 257-64. Disponible en: http:// scielo.sld.cu/pdf/rhcm/v12n2/rhcm13213.pdf.

10. Shannon K, Strathdee AS, Goldenberg SM, Duff P, Mwangi P, Rusakova M, et al. Global epidemiology of HIV among female sex workers: influence of structural determinants. Lancet [Internet]. 2015 [citado 2018 set. 04];385(9962):55-71. DOI: http:// dx.doi.org/10.1016/S0140-6736(14)60931-4.

11. Leite GS, Murray l, Lenz F. The Peer and Nonpeer: the potential of risk management for HIV prevention in contexts of prostitution. Rev Bras Epidemiol [Internet]. 2015 [citado 2018 set. 04];18 Suppl 1:7-25. DOI: http://dx.doi.org/10.1590/18094503201500050003 .

12. Beattie TS, Isac $S$, Bhattacharjee $P$, Javalkar $P$, Davey $\mathrm{C}$, Raghavendra $\mathrm{T}$. Reducing violence and increasing condom use in the intimate partnerships of female sex workers: study protocol for Samvedana Plus, a cluster randomised controlled trial in Karnataka state, south India. BMC Public Health [Internet]. 2016 [citado 2018 set. 04];16:660. DOI: http:// dx.doi.org/10.1186/s12889-016-3356-7.

13. Schwitters A, Swaminathan M, Serwadda D, Muyonga M, Shiraishi RW, Benech I, et al. Prevalence of rape and client-initiated gender-based violence among female sex workers: Kampala, Uganda, 2012. AIDS Behav [Internet]. 2015 [citado 2018 set. 04];19 Suppl 1:S68-76. DOI: http://dx.doi.org/10.1007/ s10461-014-0957-y.

14. Madeiro AP, Diniz D. Induced abortion among Brazilian female sex workers: a qualitative study. Ciênc Saúde Coletiva [Internet]. 2015 [citado 2018 set. 04];20(2):587-93. DOI: http://dx.doi. org/10.1590/1413-81232015202.11202014.

15. Oliveira TJ, Rios MA, Teixeira PN. Mortality of woman of childbearing age in the health region of Guanambi/BA. O mundo da saúde [Internet]. 2017 [citado 2018 set. 04]; 41(4):711-9. DOI: http://doi. org/10.15344/0104-7809.20174104711719.

16. Oliveira-Campos M, Nunes ML, Madeira FC, Santos MG, Bregmann SR, Malta DC, et al. Sexual behavior among Brazilian adolescents, National Adolescent School-based Health Survey (PeNSE 2012). Rev Bras Epidemiol [Internet]. 2014 [citado 2018 
set. 04];17 Suppl 1:116-30. DOI: http://dx.doi. org/10.1590/1809-4503201400050010.

17. Pitpitan EV, Kalichman SC, Eaton LA, Strathdee AS, Patterson TL. HIV/AIDS risk among venue-based female sex workers across the globe: a look back and the way forward. Curr HIV/AIDS Rep [Internet]. 2013 [citado 2018 set. 04];10(1):65-78. DOI: http://dx.doi. org/10.1007/s11904-012-0142-8.

18. Baral SD, Friedman MR, Geibel S, Rebe K, Bozhinov B, Diouf D, et al. Male sex workers: practices, contexts, and vulnerabilities for HIV acquisition and transmission. Lancet [Internet]. 2014 [cited 2018 Nov 17];385(9964):260-73. Available from: https:// www.ncbi.nlm.nih.gov/pmc/articles/PMC4504188/ pdf/nihms656919.pdf.

19. Shannon K, Goldenberg G, Deering K, Strathdee SA. HIV infection among female sex workers in concentrated and high prevalence epidemics: why a structural determinants framework is needed. Curr Opin HIV AIDS [Internet]. 2014 [cited 2018 Nov 17];9(2):174-82. Available form: https://www. ncbi.nlm.nih.gov/pmc/articles/PMC4286412/pdf/ nihms588656.pdf.

20. Pinheiro TF, Calazans GJ, Ayres JRCM. Uso da Camisinha no Brasil: um olhar sobre a produção acadêmica acerca da prevenção de HIV/Aids (20072011). Temas Psicol [Internet]. 2013 [cited 2018 Nov 17];21(03): 815-36. DOI: http://dx.doi.org/10.9788/ TP2013.3-EE07PT.

21. Nascimento SS, Garcia LG. Nas armadilhas do desejo: privações e movimentos de jovens prostitutas em zonas rurais. Cad CRH [Internet]. 2015 [cited 2018 Nov 17];28(74):383-96. DOI: http:// dx.doi.org/10.1590/S0103-49792015000200010.

22. Penha JC, Aquino CBC, Neri EAR, Reis TGO, Aquino PS, Pinheiro AKB. Risk factors for sexually transmitted diseases among sex workers in the interior of Piaui, Brazil. Rev Gaúcha Enferm [Internet]. 2015 [cited 2018 Nov 17];36(2):639. DOI: http://dx.doi.org/10.1590/19831447.2015.02.52089.

23. Araújo SL, Zazula R. Sexualidade na terceira idade e terapia comportamental: revisão integrativa. Rev Bras de Ciências do Envel Humano [Internet]. 2015 [cited 2018 Nov 17];12(2):172-82. DOI: http://doi. org/10.5335/rbceh.v12i2.5054.

24. Dourado I, MacCarthy S, Reddy M, Calazans G, Gruskin S. Revisiting the use of condoms in Brazil. Rev Bras Epidemiol [Internet]. 2015 [cited 2018 Nov 17];18 Suppl 1:63-88. DOI: http://dx.doi. org/10.1590/1809-4503201500050006.

25. Venson AM. Tráfico de pessoas para exploração sexual? Uma análise de processos-crime (19952012). Estudos Feministas [Internet]. 2017 [cited 2018 Nov 17];25(2):571-91. DOI: http://dx.doi. org/10.1590/1806-9584.2017v25n2p571.

26. Sardinha NS, Santos MIG, Bonafé SM. Estudo observacional sobre HIV/AIDS em indivíduos entre 13 e 19 anos do município de Maringá (PR). Rev Saúde e Pesquisa [Internet]. 2015 [citado 2018 Nov 04];8(1):71-8. DOI: http://dx.doi.org/10.17765/19831870.2015v8n1p71-78.

Recebido em: 24/07/2019

Aceito em: 05/09/2019 